\title{
Akupunkturnadeln können Praxiskosten darstellen
}

\begin{abstract}
_Laut der Legende der Nr. 30791 EBM sind die Kosten für Akupunkturnadeln mit dem Honorar abgegolten. Das be-
\end{abstract}

deutet, dass man als Vertragsarzt die Kosten für diese Nadeln selbst trägt man muss also zusehen, dass man über das Honorar eine ausreichende Refinanzierung erreicht. Das ist angesichts der Tatsache, dass die Akupunkturleistungen im EBM quotiert ausgezahlt werden und bei den entsprechend behandelten Patienten die Grundpauschale nach Nr. 03040 nicht gezahlt wird, keineswegs selbstverständlich.

\section{MMW-KOMMENTAR}

In der GOÄ ist das anders geregelt. Dort kann zusätzlich

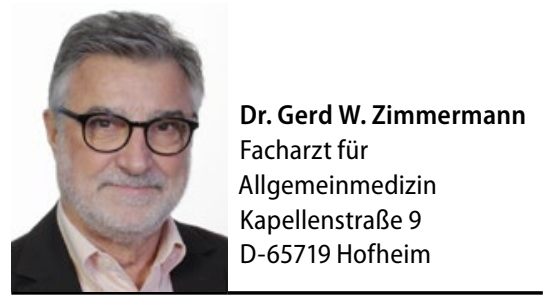

zu den Leistungen der Schmerzakupunktur nach den Nrn. 269 und 269a entsprechend $\S 10$ der GOÄ auch der Ersatz von Auslagen in Rechnung gestellt werden. Davon ausgeschlossen sind ausdrücklich nur einzeln aufgeführte Einmalmaterialien wie Einmalspritzen, Einmalskalpelle etc. Akupunkturnadeln sind in dieser abschließenden Aufzählung nicht enthalten. Folglich kann der Wert der Gesamtzahl der pro Sitzung genutzten Akupunkturnadeln zusätzlich in Rechnung gestellt werden. Es gilt auch zu bedenken, dass nur die Akupunktur bei chronischen Schmerzen der Lendenwirbelsäule oder der Kniegelenke durch Gonarthrose vertragsärztliche Leistungen darstellen. Alle übrigen Indikationen können nach GOÄ berechnet werden - sowohl bei privat Versicherten als auch bei Kassenpatienten.

Diese Akupunktur ist immer eine Privatleistung.

\section{Beim Praxisurlaub das Gesetz beachten}

_ Im $\$ 7$ des Bundesurlaubsgesetzes (BUrlG) ist bestimmt, dass bei der zeitlichen Festlegung des Praxisurlaubs die Urlaubswünsche des Arbeitnehmers zu berücksichtigen sind. Allerdings sind Ausnahmen möglich, wenn es einen Konflikt mit dringenden betrieblichen Belangen oder aber mit den Urlaubswünschen anderer Arbeitnehmer gibt, die unter sozialen Gesichtspunkten Vorrang haben - etwa wegen Schulferien, Kita-Schließzeiten etc.

$\mathrm{Zu}$ den dringenden betrieblichen Belangen zählen auch Betriebsferien. Diese können z. B. in Arztpraxen vom Arbeitgeber festgesetzt werden, wenn dieser in Urlaub fahren will und wegen
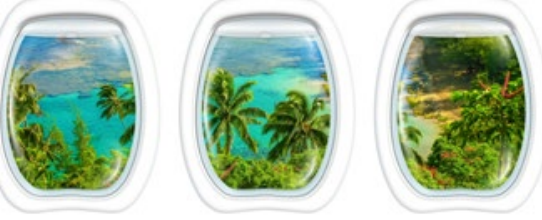

der Abwesenheit des Arztes ein Praxisbetrieb nicht mehr möglich ist. Ein anderer Grund für Betriebsruhe wäre z. B. eine Fortbildung des einzigen Arztes in der Praxis. Auch Brückentage können als Betriebsurlaub festgelegt werden.

\section{MMW-KOMMENTAR}

Betriebsferien können allerdings niemals spontan angeordnet werden, etwa weil der Praxisinhaber kurzfristig ein verlängertes
Urlaubswochenende plant. Sie müssen vielmehr bereits zu Beginn des Urlaubsjahrs der Belegschaft bekanntgegeben werden. Darüber hinaus kann die Praxis nach einer Entscheidung des Bundesarbeitsgerichts höchstens über drei Fünftel des Jahresurlaubs eines Praxisangestellten verfügen. Den Rest kann der Arbeitnehmer frei wählen. Stehen einer medizinischen Fachangestellten (MFA) beispielsweise 25 Urlaubstage zu, kann der Praxisinhaber nur 15 davon über Betriebsferien verplanen. Die restlichen 10 Tage müssen dann gewährt werden, wenn die MFA dies wünscht. Voraussetzung ist allerdings auch hier, dass dieser Wunsch nicht mit denen der anderen Praxisangestellten kollidiert. 